\title{
DSRC versus 4G-LTE for Connected Vehicle Applications: A Study on Field Experiments of Vehicular Communication Performance
}

\author{
Zhigang Xu, ${ }^{1}$ Xiaochi Li, ${ }^{1}$ Xiangmo Zhao, ${ }^{1}$ Michael H. Zhang, ${ }^{2}$ and Zhongren Wang ${ }^{3}$ \\ ${ }^{1}$ School of Information Engineering, Chang'an University, No. 435, The Middle Section of South 2nd-Ring Road, Xian, \\ Shaanxi 710064, China \\ ${ }^{2}$ Department of Civil and Environmental Engineering, University of California, Davis, 3145 Ghausi Hall, \\ One Shields Avenue, Davis, CA 95616, USA \\ ${ }^{3}$ California Department of Transportation, Division of Maintenance, Headquarters, 2389 Gateway Oaks Drive, \\ No. 200, MS 91, Sacramento, CA 958833, USA
}

Correspondence should be addressed to Xiangmo Zhao; xmzhao@chd.edu.cn

Received 14 April 2017; Accepted 3 July 2017; Published 23 August 2017

Academic Editor: Xiaobo Qu

Copyright (C) 2017 Zhigang Xu et al. This is an open access article distributed under the Creative Commons Attribution License, which permits unrestricted use, distribution, and reproduction in any medium, provided the original work is properly cited.

Dedicated short-range communication (DSRC) and 4G-LTE are two widely used candidate schemes for Connected Vehicle (CV) applications. It is thus of great necessity to compare these two most viable communication standards and clarify which one can meet the requirements of most $\mathrm{V} 2 \mathrm{X}$ scenarios with respect to road safety, traffic efficiency, and infotainment. To the best of our knowledge, almost all the existing studies on comparing the feasibility of DRSC or LTE in V2X applications use software-based simulations, which may not represent realistic constraints. In this paper, a Connected Vehicle test-bed is established, which integrates the DSRC roadside units, 4G-LTE cellular communication stations, and vehicular on-board terminals. Three Connected Vehicle application scenarios are set as Collision Avoidance, Traffic Text Message Broadcast, and Multimedia File Download, respectively. A software tool is developed to record GPS positions/velocities of the test vehicles and record certain wireless communication performance indicators. The experiments have been carried out under different conditions. According to our results, 4G-LTE is more preferred for the nonsafety applications, such as traffic information transmission, file download, or Internet accessing, which does not necessarily require the high-speed real-time communication, while for the safety applications, such as Collision Avoidance or electronic traffic sign, DSRC outperforms the 4G-LTE.

\section{Introduction}

In 2011, the US Department of Transportation (US DOT) announced plans to support the introduction of vehicle-tovehicle $(\mathrm{V} 2 \mathrm{~V})$ communication among light-duty vehicles in the USA, commonly known as "Connected Vehicles" [1-4]. Connected Vehicle focuses on localized V2V, Vehicleto-Infrastructure (V2I), and Vehicle-to-Device (V2X) Systems to support safety, mobility, and environmental applications using Dedicated Short-Range Communications (DSRC)/Wireless Access for Vehicular Environments (WAVE) [5]. A pilot study was conducted at UMTRI to examine the feasibility of $\mathrm{V} 2 \mathrm{~V}$ communication in a largescale, real-world environment [6].
Many vehicle manufacturers also pay due attention to the research and implementation of Connected Vehicle. The Crash Avoidance Metrics Partnership (CAMP) Vehicle Safety Consortium Communications (VSCC) comprising BMW, Daimler Chrysler, Ford, GM, Kia, Nissan, Toyota, and Volkswagen, in partnership with USDOT, proposed more than 57 application scenarios about Connected Vehicle, like safety applications, nonsafety applications, high potential benefit safety applications, and other applications [7]. Miao et al. [8] listed 8 safety-related applications and their latency requirements (See Table 1).

US DOT has developed a Connected Vehicle Reference Architecture (CVRA) to help guide deployment of components by road operators and automotive, highway, and 
TABLE 1: Active safety latency requirements (units: seconds).

\begin{tabular}{lc}
\hline Traffic signal violation warning & 0.1 \\
Curve speed warning & 1.0 \\
Emergency electronic brake lights & 0.1 \\
Precrash sensing & 0.02 \\
Cooperative forward collision warning & 0.1 \\
Left turn assistant & 0.1 \\
Lane change warning & 0.1 \\
Stop sign movement assistance & 0.1 \\
\hline
\end{tabular}

aftermarket equipment manufacturer and service providers [9]. In CVRA, wireless communication technology is the key and fundamental part, which directly affects the implementation, performance, reliability, and interoperation of the transportation applications.

Between DSRC and LTE, which one is an appropriate technology for Connected Vehicle applications? Will the combination or hybrid solution be more promising? This is an urgent open question which has been discussed recently.

Considering the harsh vehicular environment and related communication concerns, such as high level of the mobility of the nodes, multipath, and environmental dynamics caused by vehicles and pedestrians, IEEE proposed a modified version of the Wireless Local Area Network (WLAN) protocol, IEEE802.11p (commonly called "DSRC"), for vehiclevehicle and vehicle-infrastructure communication. A dedicated bandwidth of $75 \mathrm{MHz}$ in the 5.850 to $5.925 \mathrm{GHz}$ band has been allocated by the US Federal Communications Commission (FCC) [10]. But, to the DSRC, a key drawback is its low scalability. It lies in the fact that the protocol is unable to provide the required time-probabilistic characteristics when travelling in a dense traffic [11].

The existing cellular wireless infrastructure, particularly the $4 \mathrm{G}$-LTE, has potential to be redesigned as a communication basis for vehicular cooperative safety systems, which can offer low latencies and high throughputs simultaneously, thus enabling more bandwidth-demanding and real-time critical services for end-users [12]. Nevertheless, US DOT has been reluctant of considering cellular-based telematics over a long time as it would force car owners and state DOTs to constantly upgrade equipment in vehicles and intersections, respectively [13]. On December 13, 2016, US DOT issued a proposed rule that would advance the deployment of Connected Vehicle technologies throughout the US light vehicle fleet. The rule will mandate equipping DSRC devices on all new light-duty vehicles produced in the USA [14].

In China, there still exists a debate on whether DSRC should be utilized as the communication standard of the physical layer in the Connected Vehicle architecture of China, since the LTE networks are widely deployed all over the country and the cellular capabilities have already been on the roadmap for many vehicle manufacturers and for telematics applications [15]. Therefore, LTE-based technologies, as well as DSRC, are currently supported by different stakeholders, government departments, infrastructure vendors, and vehicle manufacturers in China [16]. In 2016, Chen et al. [17, 18] from China Academy of Telecommunication Technology proposed long-term evolution-V (LTE-V) as a systematic and integrated V2X solution based on time-division LTE (TD-LTE). Compared with DSRC, LTE-V-direct is a new decentralized architecture which modifies TD-LTE physical layer and tries to keep commonality as possible to provide short-range direct communication, low latency, and high reliability improvements. However, LTE-V is still on trial stage, and it is a long term to freeze its standardization and deploy large-scale commercial applications. In this regard, it is necessary to compare these two most viable communication standards (DSRC and 4G LTE) in terms of functionality and performance.

Many researchers have expressed considerable research interests on the vehicular networking and proposed various solutions. However, the majority of these studies are based on computer simulation to avoid the high costs in the real field experiments. Vinel [19] provides a theoretical framework which compares the basic patterns of both DSRC and LTE in the context of the safety of life vehicular scenarios. According to the numerical experiments, the author concludes that the abilities of LTE to support beaconing for vehicular safety applications are poor as the LTE network easily becomes overloaded even under the idealistic assumptions. Trichias et al. [20] analyze the feasibility of LTE technology for the Intelligent Transportation Systems (ITS). The model built in his paper simulates the Uplink operations of LTE, in a vehicular network supporting ITS. It indicates that LTE can meet the ITS requirements in terms of latency and capacity and in some cases even outperforms DSRC. Mir and Filali [21] conduct the performance evaluation of IEEE 802.11p and LTE using the ns-3 simulator. Both DSRC and LTE are compared in terms of delay, reliability, scalability, and mobility support in the context of various application requirements. The results indicate that IEEE 802.11p offers acceptable performance for sparse network topologies with limited mobility support. On the other hand, LTE meets most of the application requirements regarding reliability, scalability, and mobility support. However, LTE is challenging when obtaining stringent delay requirements in the presence of higher cellular network traffic load. There is still a lack of practical measurement between DSRC and LTE for developing vehicular applications in the open literature.

Compared with field testing, the software-based protocol simulation testing has the advantages of low cost, short deployment cycle, and flexible parameters setting. The shortcomings are also obvious: (1) the mathematical models used in a software-based simulation is formulated in an idealistic environment. The signal attenuations caused by vehicles, surrounding buildings, or vegetation are neglected; (2) the differences among the communication equipment and user terminals such as the performance diversity and installation conditions are hardly considered; and (3) the results of a software-based simulation are generally better than the actual field tests. However, in the practical application environment, these results will degrade to some extent.

1.1. Research Objectives. On the basis of the above literature survey, it can be confirmed that it is greatly necessary to test 
the function and performance of DSRC and LTE in a real road environment, so as to verify the simulation results of various theoretical models and provide important data for the development of various vehicular networking applications.

This study tests the communication quality and reliability of the DSRC and LTE in a real road environment under 3 classic Connected Vehicle scenarios: Collision Avoidance, Traffic Message Broadcast, and Multimedia File Download between vehicle and infrastructure. This can be a critical baseline for many other Connected Vehicle applications. This paper made 3 key contributions. First, we build an independent and private wireless network platform made up of DSRC and LTE in Chang'an University Cooperative Vehicle and Infrastructure System (CU-CVIS) test-bed. This platform integrates the 4G-LTE cellular stations and the core network, DSRC roadside units, and vehicular on-board terminals. With the platform, lots of vehicular communication experiments under different mobility conditions can be conducted. Second, three Connected Vehicle application scenarios are set as Collision Avoidance, Traffic Text Message Broadcast, and Multimedia File Download, respectively. A software tool is developed to record the GPS-based position and velocity of the vehicles that are moving on the testbed. With the tool, some crucial wireless communication performance indicators of DSRC and 4G-LTE such as the throughput, data loss rate, and the latency time under the above scenarios are acquired. Third, the results of the onfield vehicular communication performance are analyzed. The suitability of DSRC and 4G-LTE on the most popular Connected Vehicle scenarios is discussed. Moreover, some suggestions are given on how to integrate these two kinds of technologies in the future Connected Vehicle applications.

This paper is organized as follows. Section 2 presents the architecture of the CU-CVIS test-bed and deployment scheme of the DSRC and LTE wireless network platform. Section 3 presents the setting of the experiment. Section 4 presents the experimental data and discussion on the comparison of DSRC and LTE. The paper ends with some concluding remarks in Section 5.

\section{Construction of Vehicular Communication Platform}

As shown in Figure 1, a vehicular communication platform in CU-CVIS (Changan University Cooperative VehicleInfrastructure System) test-bed is built. CV-CVIS is located in Weishui Campus of Chang'an University, which occupies 282,000 square meters (about 70 acres). It includes a 2.4kilometer high-speed circular test road with 2 lanes and an extra 1.1-kilometer straight 4-lane test track with 4 kinds of pavement (asphalt, concrete, bricks, and dirt). It is a comprehensive and closed environment for the testing of various Connected Vehicle applications. It is made up of 4 parts (see Figure 1(b)), which are smart roadsides, connected and automated vehicles, heterogeneous network, and transportation applications severs, respectively.

On the test-bed, we install a short-range wireless system with 4 DSRC roadside units (RSUs) and a 4G-LTE cellular communication system, which comprise a specialized vehicular communication platform. Both DSRC and LTE systems are isolated with the public telecom network. The locations of the RSUs and LTE antennas are denoted in Figure 3.

The DSRC RSUs are mounted on the gantries established on the test-bed (see Figure 2). The core part of the RSU is the module WBOX1001, which is developed by China Genvict Tech Co., Ltd. The module utilizes a high-performance ARM chip NXP SAF5100 as the processor, which is running IEEE $802.11 \mathrm{p}$ and IEEE1609 protocol stacks. The working frequency is $5.850 \mathrm{GHz}-5.925 \mathrm{GHz}$ with a data rate up to $27 \mathrm{Mb} / \mathrm{s}$. The maximal communication distance is 300 meters under the default transmitting power. The maximal latency time is 150 milliseconds with a 4096-bit package.

The 4G-LTE system is a private cellular wireless network operating on the frequency of $1.88-1.9 \mathrm{GHz}$ with TDLTE protocol, which is developed by China Datang Mobile Co., Ltd. As shown in Figure 3 it comprises of eNode-B base station, Customer Premise Equipment (CPE) terminals, Evolved Packet Core (EPC), servers cluster, and switches. Their functions are described as follows:

(1) OBU connects LTE network through CPE, which realizes LTE protocol stack and TCP/IP protocol stack by wireless or wired mode and transmits data to the destination through $4 \mathrm{G}$ network.

(2) eNode-B is made up of RRU and BBU, which is a terminal of the air interface protocol and the first node to contact with user equipment (UE). eNode$B$ is responsible for the wireless bearer, downlink dynamic wireless resources, data packet scheduling, and mobility management.

(3) EPC is made up of Serving Gateway (SGW), PDN Gateway (PGW), Mobility Management Entity (MME), and Policy and Charging Rules Function (PCRF), which is responsible for data exchange and processing.

The application servers play very important roles, which are used for data collection, storage, and processing, and are connected to the base station through Gigabit Ethernet. The LTE platform has 4 directional antennas with the maximal transmission power of $60 \mathrm{w}$, which realize the full wireless signal coverage of the whole test-bed. The LTE platform is divided into 4 cells. Each cell can accommodate up to 200 items of UE with Uplink rate of $20 \mathrm{Mb} / \mathrm{s}$ and downlink rate of $80 \mathrm{Mb} / \mathrm{s}$.

The constructed vehicular communication platform can conduct 4 kinds of wireless communication experiments: (1) V2V via DSRC; (2) V2V via LTE; (3) V2I via DSRC; (4) V2I via LTE. There are many famous test-beds around the world for testing connected and automated vehicles, such as the MCity of the University of Michigan and the GoMento test site located in Contra Costa County, California. These two test-beds focus on demonstration testing for applications of future Intelligent Transportation Systems, which mainly aims to verify the integrity performance and data-flow logic of the applications developed for the connected and automated vehicles (CAVs). CU_CVIS test-bed of Chang'an University 


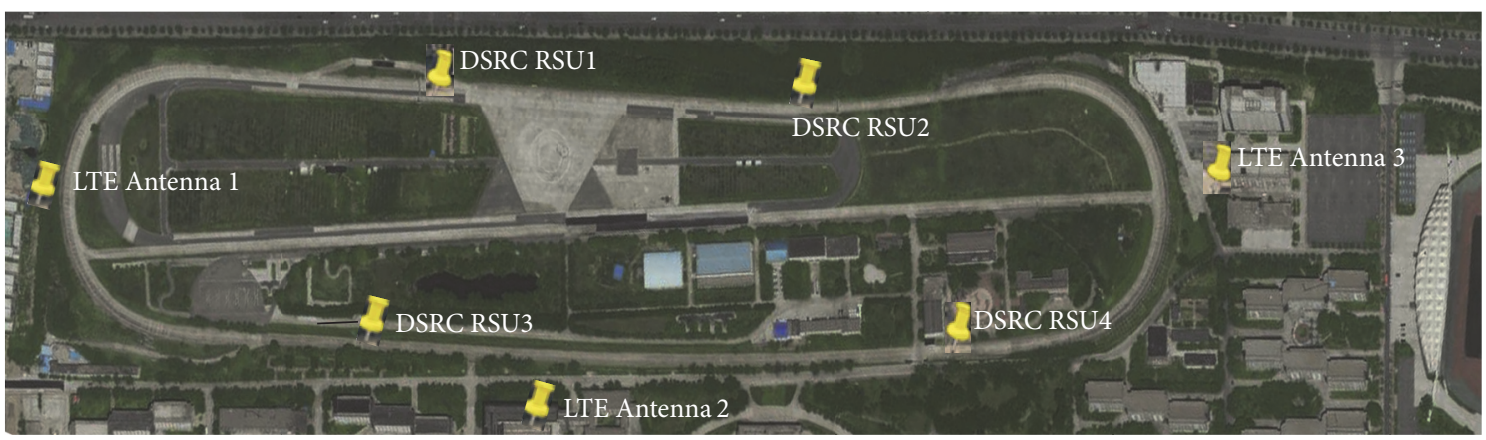

(a) The bird-view satellite image of CU-CVIS test-bed

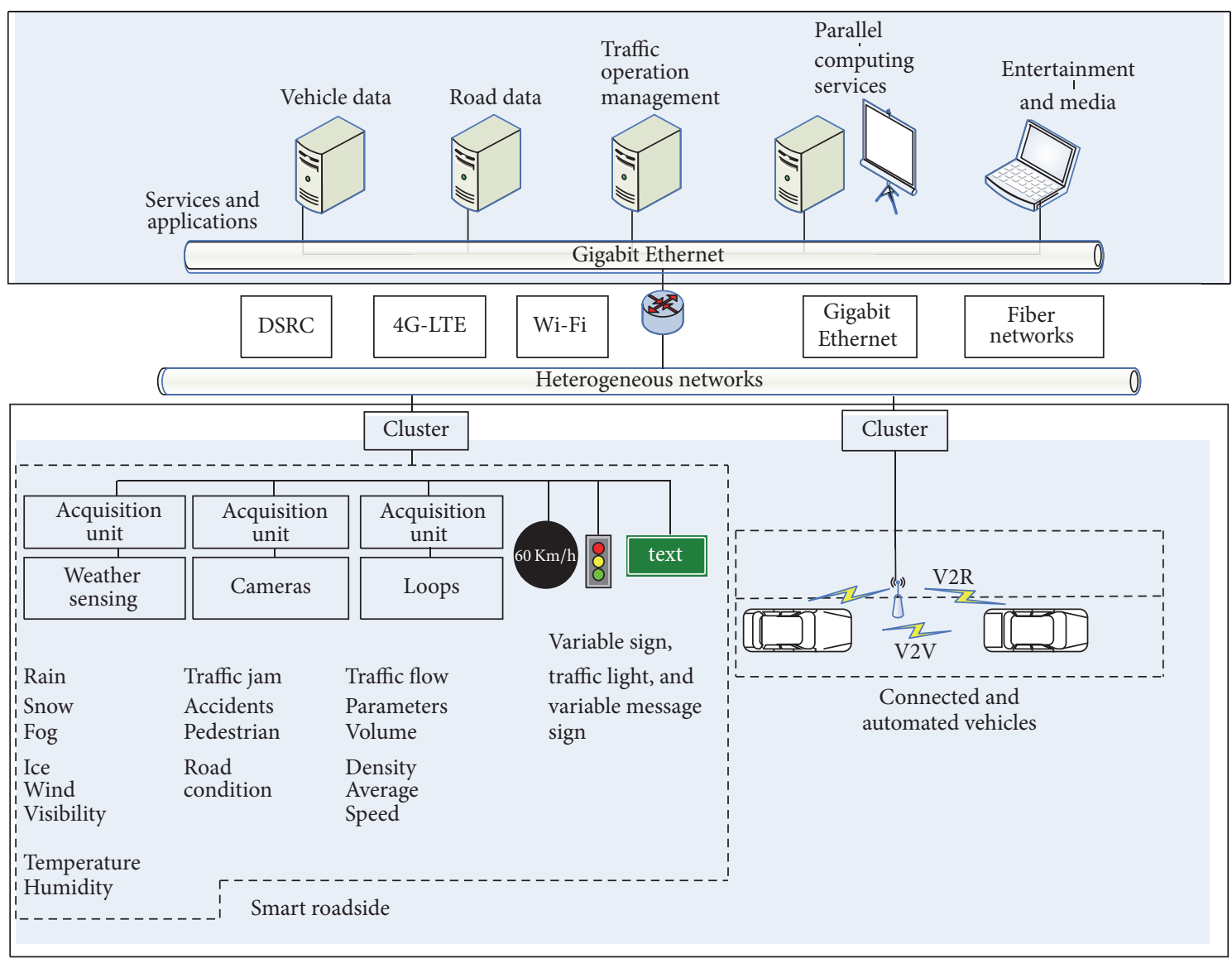

(b) The logic architecture of CU-CVIS test-bed

Figure 1: Chang'an University Cooperative Vehicle-Infrastructure System test-bed.

focuses on the metafunction testing for each part of CAVs and the comprehensive performance testing under the limit conditions. For instance, CU_CVIS can test the performance of different communication modes and verify whether these communication methods can meet the requirements of some intelligent transportation applications on the real time and reliability of data transmission. It can test some fundamental functions of CAVs, such as positioning accuracy, target recognition accuracy based on vision, vehicle lateral or longitudinal control ability in high-speed condition, complex environment, and bad weathers.

\section{Experimental Setup}

3.1. Setup of the On-Board Devices. We have two midsize vehicles to carry out the experiment. The setup of the onboard devices is shown in Figure 4(a). A computer with an Intel Core 2 DuoP8600 is fixed on each of the vehicles. A GPS receiver is connected to the computer via RS-232 serial interface. A LTE CPE manufactured by Datang Mobile Co., Ltd., and a DSRC Wavebox developed by Genvict Co., Ltd., are linked to the computer via Ethernet interfaces. Three kinds of antennas are mounted on the roof of the vehicles 


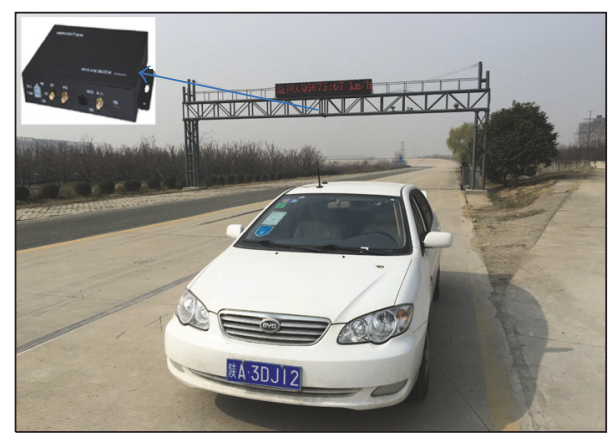

FIgURE 2: The DSRC RSU on the test-bed.

(see Figure 4(b)), they are connected to LTE terminal, DSRC terminal, and GPS module respectively. Each vehicle can talk with the other via 2 kinds of network within the coverage area. The DSRC RSU and LTE eNode-B can also send text message or digital file to the vehicle through the background servers.

3.2. Development of the Testing Software. We develop a testing software kit to test the dynamic performance of DSRC and LTE. The software kit encrypts the API functions from the device manufacturer, which can achieve the transmission of packages or files between the vehicles and roadside devices. The software kit enables 3 functions. The first is to synchronize the on-board units with the GPS clock time. The second function is to calculate the communication parameters using the encrypted APIs. For instance, the Round-Trip Time (RTT) is acquired based on the Ping function supplied by Linux OS, the Packet Loss Rate is calculated through the success rate of the transmission of the WSMP packages, and the throughput is computed on the counts of the received UDP packages during a specified time segment. The last function is to record the GPS data of the vehicles, including longitude, latitude, and velocity, which is used to analyze the effect of the distance and movement on the wireless communication performance. The software interfaces are shown in Figure 5. (a) is the client interface and (b) is the server interface.

3.3. Scenario Design. We distill 3 general scenarios from the 75 safety applications proposed by CAMP [22] to conduct the comparison experiments on the communication performance of the DSRC and LTE, which are Collision Avoidance, Traffic Text Message Broadcast, and Multimedia File Download, respectively. The weather condition of the experiments is sunny with the temperature of $25^{\circ} \mathrm{C}$ and the humidity of $50 \%$. The wind speed is $10 \mathrm{Km} / \mathrm{h}$.

3.3.1. Collision Avoidance. In this scenario (see Figure 6), the emergency Car A broadcasts the status packages to the following Car B via DSRC or LTE network at a frequency of $10 \mathrm{~Hz}$. The package size is 100 bytes, including vehicle ID, package ID, package type, package time tag, GPS data, and vehicle kinematic parameters. This scenario can also be extended to many safety applications such as approaching emergency vehicle warning, emergency electronic brake light [23-28], and platoon, The gap between Car A and Car B is controlled by the Gipps minimal safety distance model [29], which helps the following car predict the optimal speed based on current gap, velocities, and maximal acceleration (deceleration) of the cars. To ensure the usability and reliability of testing results of this scenario, the two cars run on the circular test road for 5 times at different speeds, which are $30 \mathrm{Km} / \mathrm{h}$, $60 \mathrm{Km} / \mathrm{h}, 90 \mathrm{Km} / \mathrm{h}$, and $120 \mathrm{Km} / \mathrm{h}$, respectively. Under each speed condition, we only analyze the testing data within the effective communication distance.

3.3.2. Traffic Text Message Broadcast. In this scenario (see Figure 7), when the vehicles are passing by the DSRC RSU or entering the LTE cell, the traffic information releasing system will frequently send short text messages to the vehicles. The text messages generally include the information of weather, road condition, traffic flow, work zone, accidents, travel service, and so forth. This scenario can also be extended to the applications of electronic traffic signs extension and mobile advertising push. This scenario hires a server to control the DSRC RSU and LTE to broadcast text messages to the around environment at a frequency of $10 \mathrm{~Hz}$, and the size of each message is less than 340 bytes. The on-board units installed on the passing vehicles will record not only the message content but also the affiliated information including the message ID, source ID, and time tag. The two cars will run on the circular test road for 5 times at different speeds, which is the same as in Scenario I.

3.3.3. Multimedia File Download. In this scenario, when the vehicles are passing by the DSRC RSU or entering the LTE cell, the OBUs will request file download. This scenario can also be extended to the applications of map download, Video on Demand (VOD), and so forth. In this scenario, we only employ one car to conduct the testing in order to avoid the two cars' competition for the wireless bandwidth. Once the car enters the effective communication area, it will send a download request to the server. If the request is accepted, the downloading starts and the $\mathrm{OBU}$ begins to record the throughput of the link. The running model of the car is the same as the above two scenarios. In order to ensure the reliability of the testing data, the testing cars will run on the circular test road for 5 times at different speeds to execute the application of Multimedia File Download.

\section{Experimental Results and Analysis}

4.1. Collision Avoidance. Figure 8 shows the fading characteristic of DSRC and LTE network as the velocity of the testing vehicle increases under Scenario I. Figure 8(a) shows the relationship between the average Packet Loss Rate (PLR) and the vehicle running velocity. The average PLR is calculated by the rate of the lost packages among the total sent packages. From Figure 8(a), it can be found that the average PLR of LTE jumps from $4 \%$ to $7.5 \%$ when the velocity of vehicles changes from $60 \mathrm{Km} / \mathrm{h}$ to $90 \mathrm{Km} / \mathrm{h}$, while the average PLR of DSRC changes slightly. Figure 8(b) shows the relationship 


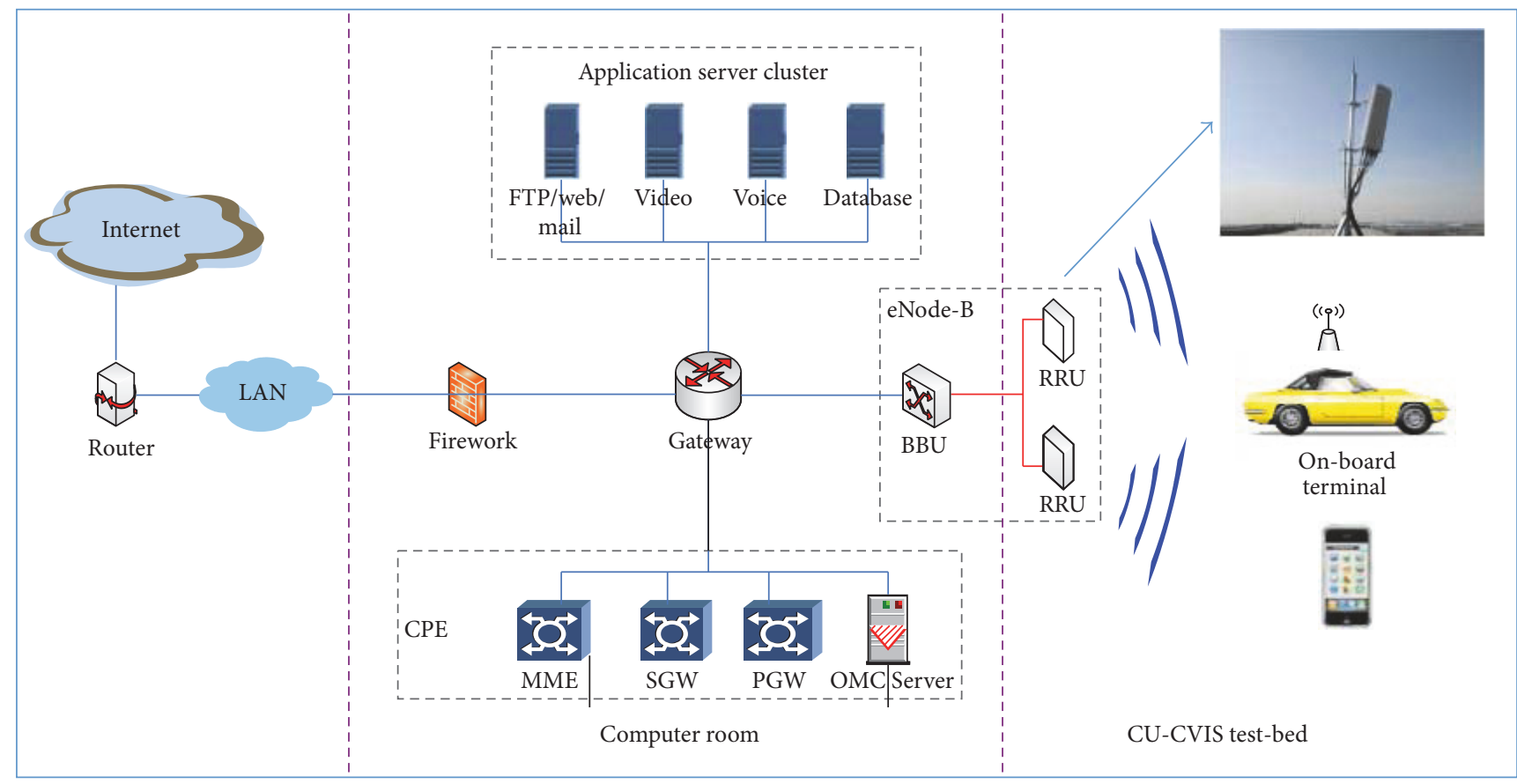

FIgURE 3: The LTE network deployed on the test-bed.

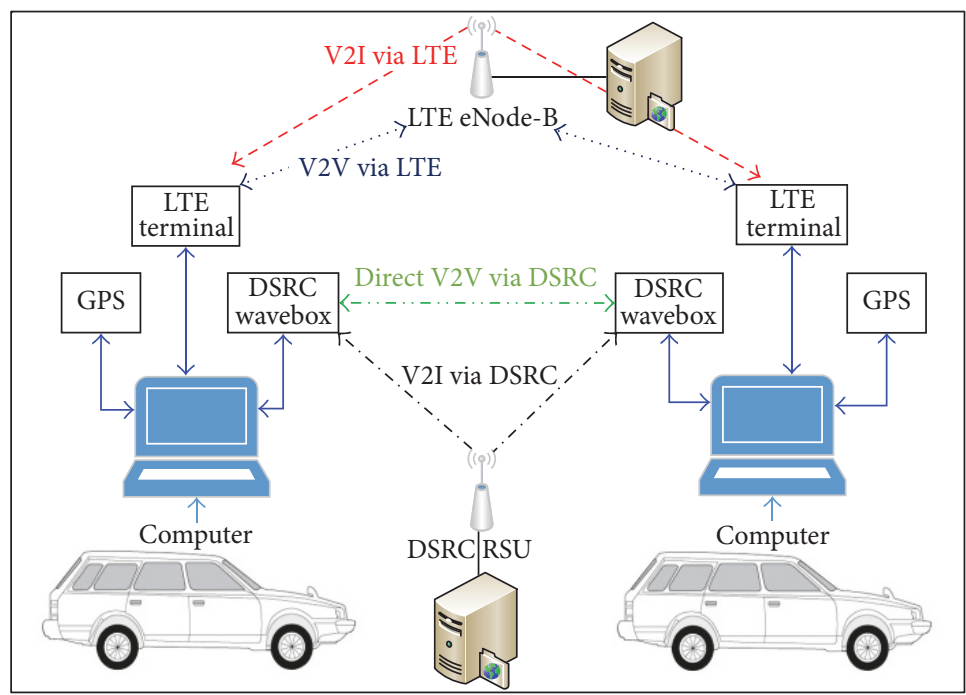

(a) The connection of on-board devices

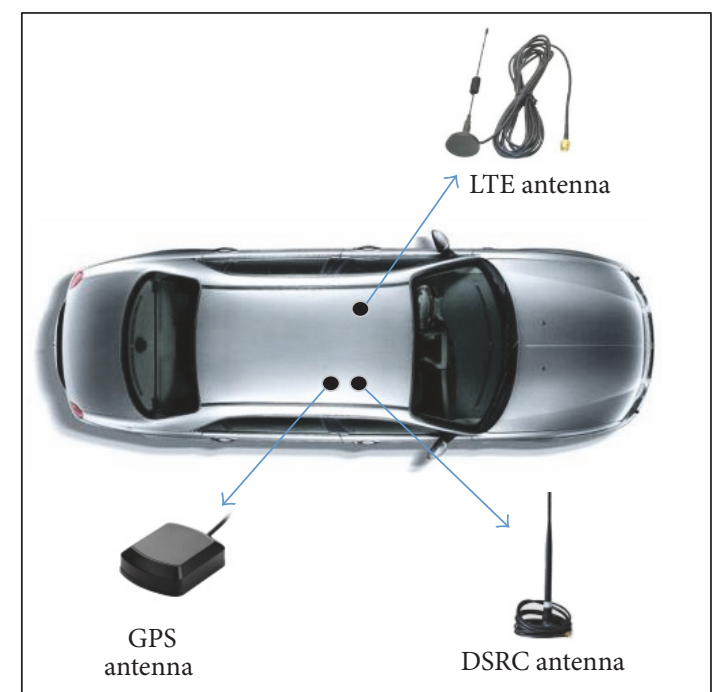

(b) The mounting positions of the antennas

FIgURE 4: The setup of the on-board devices.

between the Round-Trip Time (RTT) and the vehicle running velocity. The average RTT is calculated by the response of Ping command. From Figure 8(b), it can be found that the average RTT of DSRC is very low (below 10 milliseconds) and satisfies the most critical requirement in Table 1. But the average RTT of LTE at different vehicle speeds is much higher than that of DSRC. It is also higher than 100 milliseconds, which is the regular demand in Table 1 for safetyrelated applications. The RTT of LTE increases gradually as the vehicle speed increases, while that of DSRC keeps stable.

As can be seen in Figure 8, it can be concluded that performance degradation of LTE is worse than that of DSRC in Scenario I, which is mainly caused by Doppler Effect and cellular handoff of LTE network. In realistic case of Collision Avoidance, the latency would be higher than the measured results we got from the test because of the dense traffic flow and hash communication environment. In summary, DSRC 


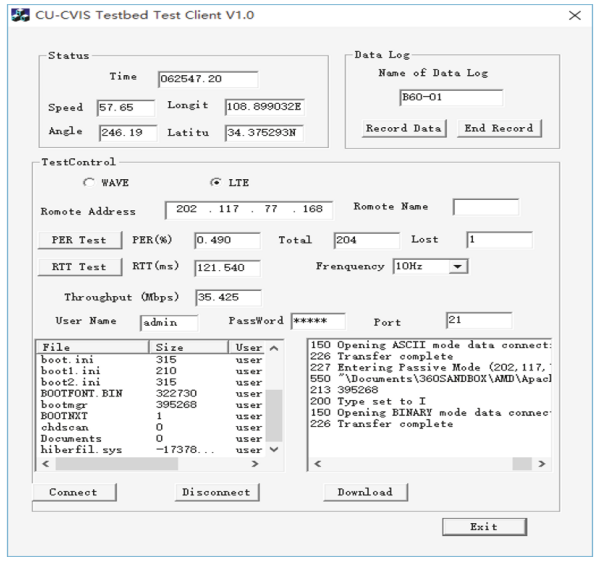

(a) The client interface

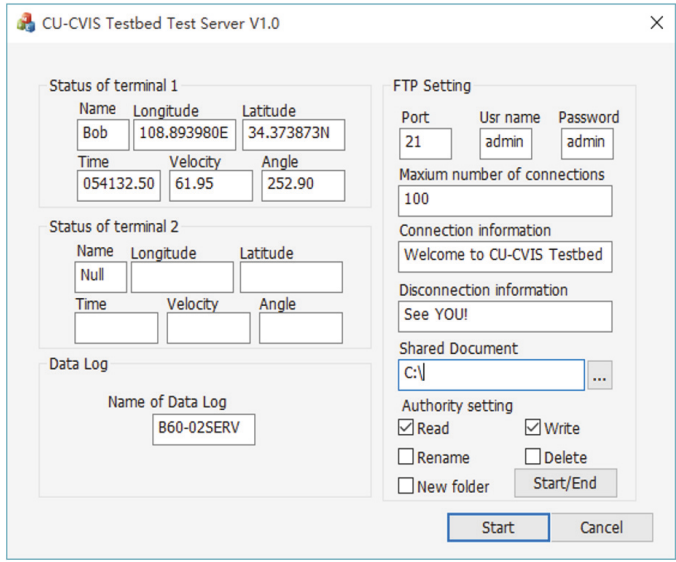

(b) The server interface

Figure 5: The developed testing software.

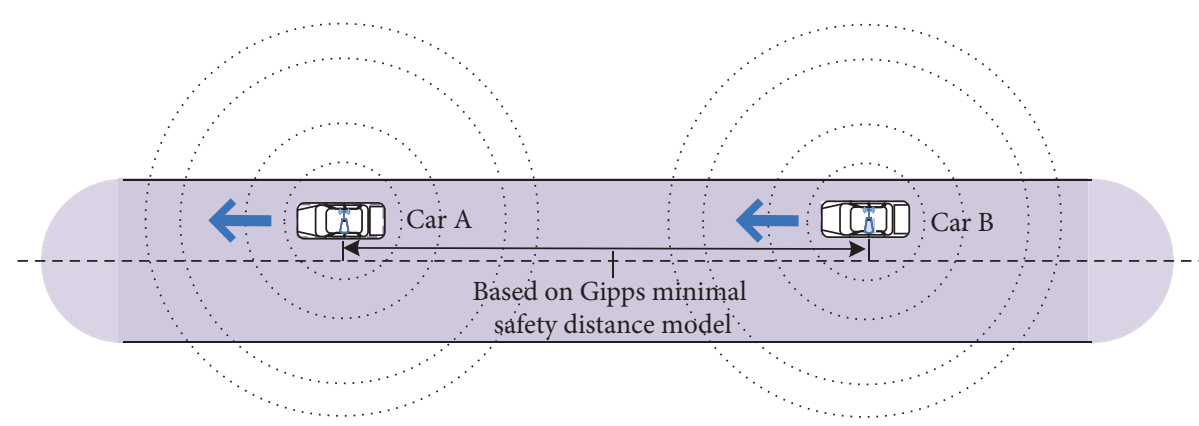

Figure 6: Collision Avoidance scenario.

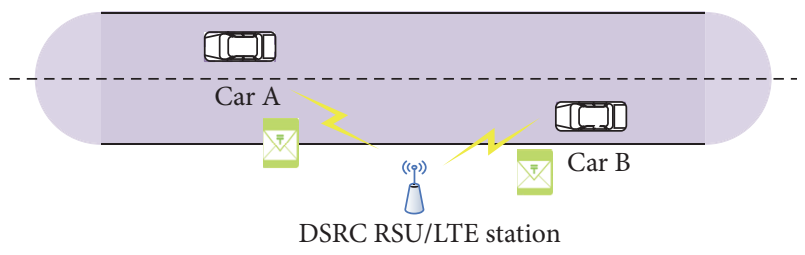

Figure 7: Traffic Text Message Broadcast scenario.

is more suitable for Collision Avoidance and other related safety V2V traffic applications.

4.2. Traffic Text Message Broadcast. Figure 9 shows the performance degradation of DSRC as the vehicle is passing by the DSRC RSU at a speed of $120 \mathrm{Km} / \mathrm{h}$, which is used to confirm the effective communication range for DSRC RSU. We can clearly observe that the average PLR and RTT abruptly begin to increase when the absolute distance between vehicle and RSU is longer than 300 meters, which is particularly evident in the Packet Loss Rate. When the distance between vehicle and RSU is more than $450 \mathrm{~m}$, it is too long for terminal on the vehicle to keep connected. Because LTE has a long coverage range while DSRC has not. We must conduct the performance comparison experiments within the effective communication range of both. Figure 9(a) shows the relationship between the Packet Loss Rate (PER) and the distance of vehicle to RSU. Figure 9(b) shows the relationship between the RoundTrip Time (RTT) and the distance. From this figure, it can be clearly observed that the PLR and RTT delay of DSRC dramatically drop off when the distance between the vehicle and RSU is close to 300 meters. So we only analyze the data captured within this range.

Figure 10 shows the of DSRC and LTE network as the velocity of the testing vehicle increases in Scenario II. Figure 10(a) shows the relationship between the average Packet Loss Rate (PLR) and the vehicle running velocity. The average PLR is calculated by the rate of the lost packages among the total sent packages. From Figure 10(a), it can be found that the average PLR of LTE jumps from $1.7 \%$ to $3.5 \%$ when the velocity of vehicles changes from $30 \mathrm{Km} / \mathrm{h}$ to $90 \mathrm{Km} / \mathrm{h}$, while the average PLR of DSRC is almost unchanged. Figure 10(b) shows the relationship between the Average Round-Trip Time (RTT and the vehicle running velocity. The average RTT is calculated by the mean of the RTT of all the broadcasted packages. From Figure 10(b), it can be found that the RTT of DSRC is very low (below 10 milliseconds, and the average RTT of LTE at $30 \mathrm{Km} / \mathrm{h}$ is lower than 100 milliseconds, which is the lowest requirement proposed by CAMP for electronic traffic sign application. When the vehicle running velocity 


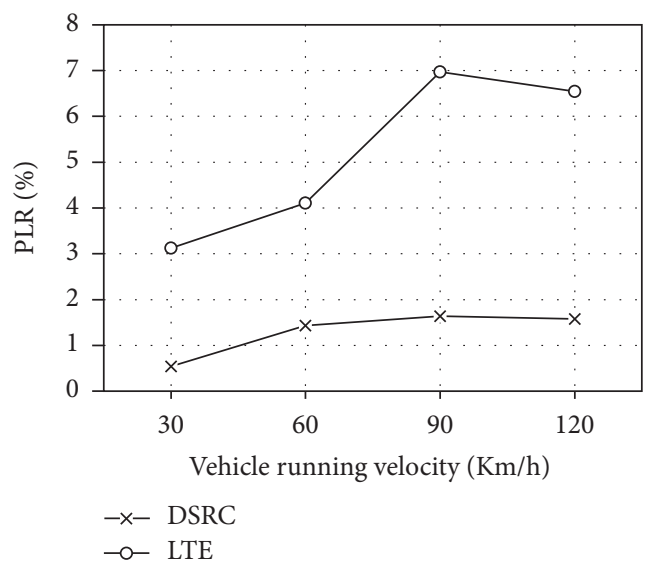

(a) PLR versus the vehicle running velocity

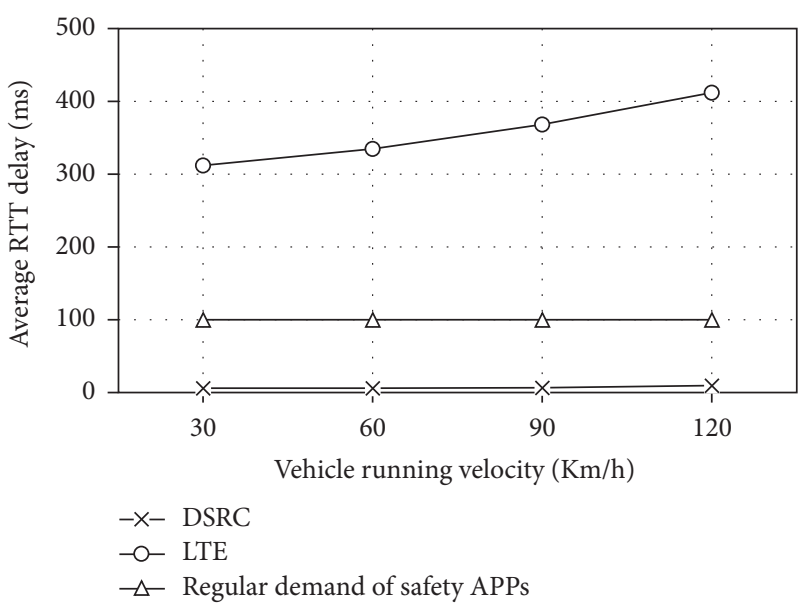

(b) Average RTT delay versus the vehicle running velocity

FIGURE 8: The performance degradation of DSRC and LTE under Scenario I.

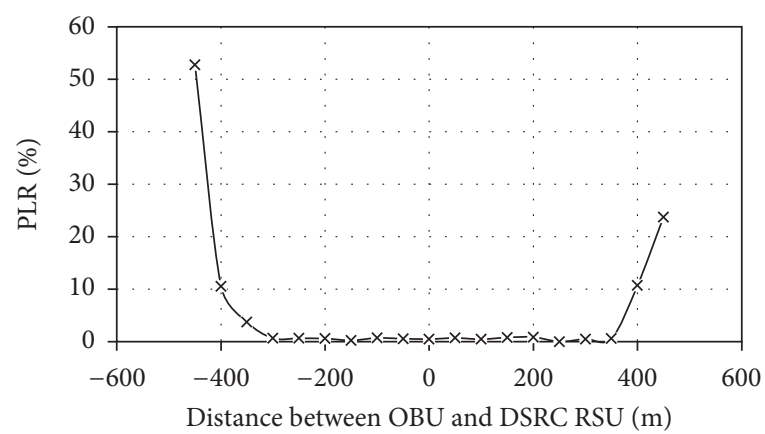

(a) PLR versus the distance between OBU and DSRC RSU

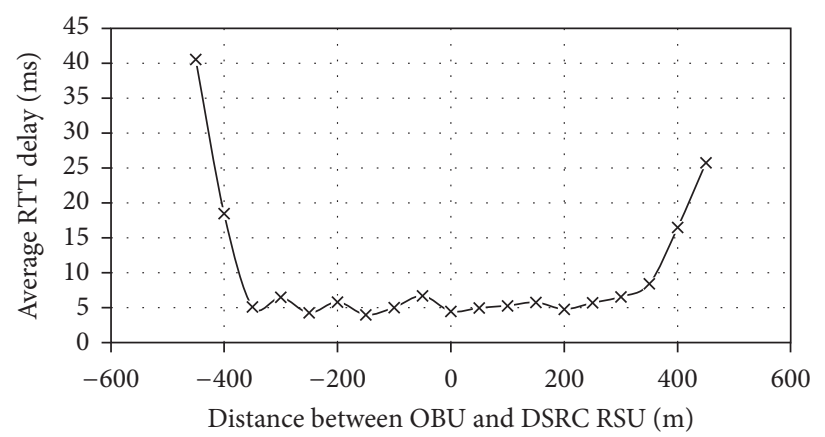

(b) Average RTT delay versus the distance between OBU and DSRC RSU

FIGURE 9: The DSRC performance versus different communication distance when the vehicle velocity is $120 \mathrm{Km} / \mathrm{h}$.

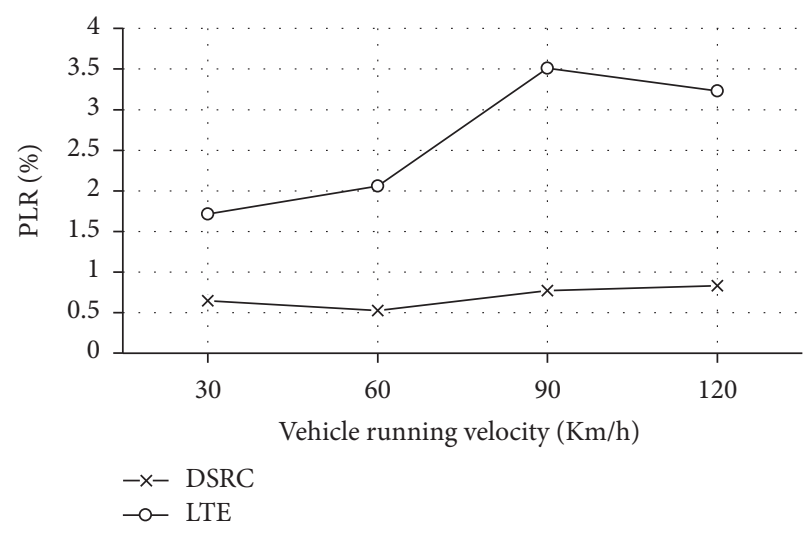

(a) PLR of both DSRC and 4G-LTE versus the vehicle running velocity

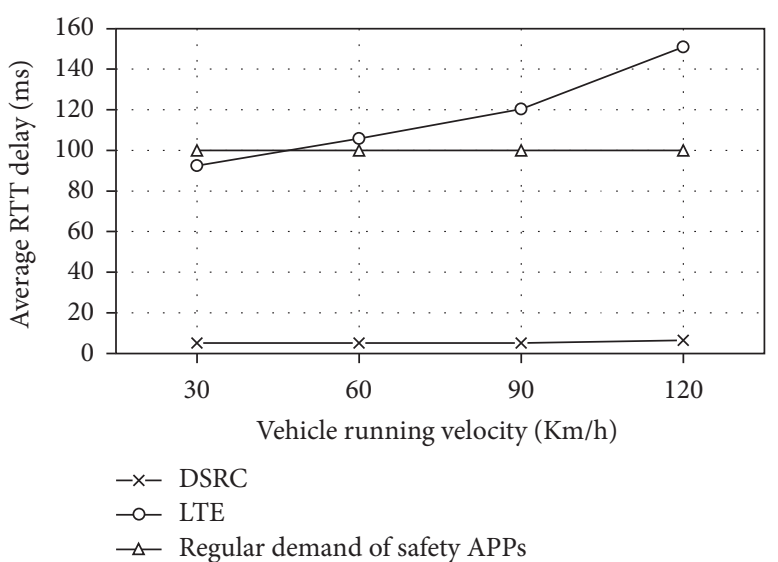

(b) Average RTT delay of both DSRC and 4G-LTE versus the vehicle running velocity

FIgURE 10: The performance degradation of DSRC and LTE under Scenario II.

exceeds $50 \mathrm{Km} / \mathrm{h}$, the average RTT of LTE is beyond 100 milliseconds and achieves 150 milliseconds at $120 \mathrm{Km} / \mathrm{h}$. The communication performance of DSRC is nearly irrelevant to the vehicle running velocity under Scenario II.
From Figure 10, it can be concluded that if the Traffic Text Message Broadcast is for the nonsafety applications, both LTE and DSRC are acceptable. And LTE has a longer coverage scope than DSRC, which helps to cut the costs of dense 


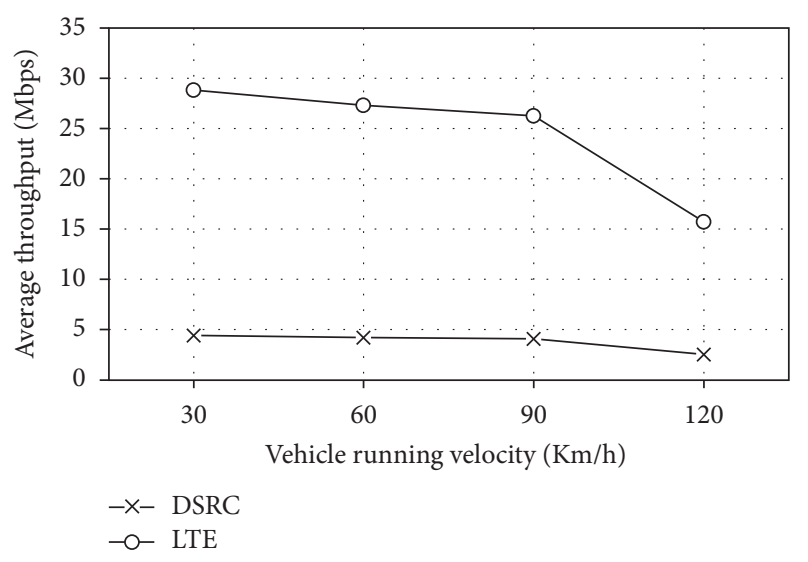

FIGURE 11: The throughput performance of DSRC and LTE in Scenario III.

deployments of DSRC RSU. But, for the safety application such as wrong way driver warming, LTE cannot satisfy the requirement.

4.3. Multimedia File Download. Figure 11 shows the throughput performance of DSRC and LTE in the scenario of Multimedia File Download. It is found that the throughput of LTE is significantly higher than that of DSRC at different vehicle running velocities although the Doppler Effect reduces their performance at high-speed conditions. Furthermore, DSRC is a kind of ad hoc network, and its throughput performance will significantly drop as the number of the nodes increases rapidly because of the traffic congestion. At the same time, LTE has a long coverage range, and it means that the vehicle equipped with LTE terminal can download the file for longer time than the ones with DSRC OBU. It is very important for download a file with big size. Besides, WAVE is a kind of ad hoc network, which makes the throughput reduced while the number of terminals increases in certain area. So in real scenarios throughput performance of WAVE may not be as good as that which we obtain in the test. Hence, it can be concluded that LTE is more suitable for Multimedia File Download scenario than DSRC.

\section{Conclusions}

In this paper, three Connected Vehicle application scenarios are set as Collision Avoidance, Traffic Text Message Broadcast, and Multimedia File Download, respectively. The communication performance of DSRC and LTE is investigated and analyzed with the developed hardware and software platform. Compared with the traditional computer simulation method for vehicle network performance, there are several innovations as follows:

(1) The performance of LTE is worse than that of DSRC under Collision Avoidance scenario, which is mainly caused by Doppler Effect and cellular handoff of LTE network. It means that LTE cannot meet the lowest requirement of 100 milliseconds for the safety applications. DSRC is more suitable for Collision Avoidance and other safety-related V2V traffic applications.

(2) Under the scenario of Traffic Text Message Broadcast, LTE has a long coverage range while DSRC has not. But, within the effective communication range, DSRC has better communication performance than LTE. Both LTE and DSRC are acceptable for the broadcast of the nonsafety text message. For the safety application such as the message broadcast from an electronic traffic sign, DSRC outperforms LTE. Because of the high costs of the dense deployment of DSRC RSUs, it is suggested that DSRC RSU can be installed on the spots where it is strongly related to safety.

(3) Under the scenario of Multimedia File Download, the throughput performance of LTE is significantly higher than that of DSRC at different vehicle running velocities. Furthermore, LTE has a long coverage range, which is more suitable for Multimedia File Download than DSRC.

(4) The combination of DSRC and LTE should be a good solution for Connected Vehicles. It not only enables the safe driving but also can supply high-quality telematics service to the drivers.

(5) In the future, the comparison experiments with more vehicles need to be conducted, because it is closer to the real-world scenarios and can test communication performance of DRSC and LTE in the extreme conditions.

\section{Conflicts of Interest}

The authors declare that there are no conflicts of interest regarding the publication of this paper.

\section{Acknowledgments}

The research is supported by the National Natural Science Foundation of China (no. 51278058), the Fundamental Application Research Program of China Ministry of Transport (no. S2013JC9397), the 111 project (no. B14043) and the Joint Laboratory of Internet of Vehicles sponsored by Ministry of Education and China Mobile. The authors also thank the anonymous reviewers and the editor for their valuable comments.

\section{References}

[1] P. J. Jin, D. Fagnant, A. Hall, and C. M. Walton, Policy Implications of Emerging Vehicle and Infrastructure Technology, 2014.

[2] F. Zhou, X. Li, and J. Ma, "Parsimonious shooting heuristic for trajectory design of connected automated traffic part I: theoretical analysis with generalized time geography," Transportation Research Part B: Methodological, vol. 95, pp. 394-420, 2017.

[3] M. Zhou, X. Qu, and S. Jin, "On the impact of cooperative autonomous vehicles in improving freeway merging: a modified 
intelligent driver model-based approach," IEEE Transactions on Intelligent Transportation Systems, vol. 18, no. 6, pp. 1422-1428, 2017.

[4] J. Ma, X. Li, F. Zhou, J. Hu, and B. B. Park, "Parsimonious shooting heuristic for trajectory design of connected automated traffic part II: computational issues and optimization," Transportation Research Part B: Methodological, vol. 95, pp. 421-441, 2017.

[5] K. C. Dey, A. Rayamajhi, M. Chowdhury, P. Bhavsar, and J. Martin, "Vehicle-to-vehicle (V2V) and vehicle-to-infrastructure (V2I) communication in a heterogeneous wireless network - Performance evaluation," Transportation Research Part C: Emerging Technologies, vol. 68, pp. 168-184, 2016.

[6] B. Schoettle and M. Sivak, "A survey of public opinion about connected vehicles in the U.S., the U.K., and Australia," in Proceedings of the 3 rd International Conference on Connected Vehicles and Expo (ICCVE '14), pp. 687-692, November 2014.

[7] C. Campolo and A. Molinaro, "Multichannel communications in vehicular Ad Hoc networks: A survey," IEEE Communications Magazine, vol. 51, no. 5, pp. 158-169, 2013.

[8] L. Miao, K. Djouani, B. J. Van Wyk, and Y. Hamam, "Performance evaluation of IEEE 802.11p MAC protocol in VANETs safety applications," in Proceedings of the IEEE Wireless Communications and Networking Conference (WCNC '13), pp. 16631668, April 2013.

[9] R. L. Bertini, H. Wang, and K. Carstens, "Preparing oregon for connected vehicle deployment," Transportation Research Record: Journal of the Transportation Research Board, vol. 2615, pp. 1-10, 2017.

[10] M. Whaiduzzaman, M. Sookhak, A. Gani, and R. Buyya, "A survey on vehicular cloud computing," Journal of Network and Computer Applications, vol. 40, no. 1, pp. 325-344, 2014.

[11] S. Lee and A. Lim, "An empirical study on ad hoc performance of DSRC and Wi-Fi vehicular communications," International Journal of Distributed Sensor Networks, vol. 9, pp. 1-10, 2013.

[12] G. Araniti, C. Campolo, M. Condoluci, A. Iera, and A. Molinaro, "LTE for vehicular networking: a survey," IEEE Communications Magazine, vol. 51, no. 5, pp. 148-157, 2013.

[13] S. Lee and A. Lim, "Reliability and performance of IEEE 802.11n for vehicle networks with multiple nodes," in Proceedings of the International Conference on Computing, Networking and Communications (ICNC '12), pp. 252-256, February 2012.

[14] NHTSA, "Proposed rule would mandate vehicle-to-vehicle (V2V) communication on light vehicles, allowing cars to "talk" to each other to avoid crashes," 2016, https://www.nhtsa.gov/ press-releases/us-dot-advances-deployment-connected-vehicletechnology-prevent-hundreds-thousands.

[15] C. Wang and M. Deng, "Promoting TD-LTE technology to V2X applications to improve traffic safety and efficiency," Modern Science \& Technology of Telecommunications, vol. 9, pp. 40-46, 2014.

[16] J. Lee, Y. Kim, Y. Kwak et al., "LTE-advanced in 3GPP Rel -13/14: An evolution toward 5G," IEEE Communications Magazine, vol. 54, no. 3, pp. 36-42, 2016.

[17] S. Chen and J. Zhao, "The requirements, challenges, and technologies for $5 \mathrm{G}$ of terrestrial mobile telecommunication," IEEE Communications Magazine, vol. 52, no. 5, pp. 36-43, 2014.

[18] S. Chen, J. Hu, Y. Shi, and L. Zhao, "LTE-V: a TD-LTE-based V2X solution for future vehicular network," IEEE Internet of Things Journal, vol. 3, pp. 997-1005, 2016.
[19] A. Vinel, “3GPP LTE versus IEEE 802.11p/WAVE: which technology is able to support cooperative vehicular safety applications?" IEEE Wireless Communications Letters, vol. 1, no. 2, pp. 125-128, 2012.

[20] K. Trichias, J. L. Berg, G. J. Heijenk, J. Jongh, and R. Litjens, "Modeling and evaluation of LTE in intelligent transportation systems," in Proceedings of the Joint ERCIM eMobility and MobiSense Workshop, University of Bern, Santorini, Greece, 2012.

[21] Z. H. Mir and F. Filali, "LTE and IEEE 802.11p for vehicular networking: a performance evaluation," EURASIP Journal on Wireless Communications and Networking, no. 89, pp. 1-15, 2014.

[22] CAMP Vehicle Safety Communications Consortium, Vehicle Safety Communications Project: Task 3 Final Report: Identify Intelligent Vehicle Safety Applications Enabled by DSRC, National Highway Traffic Safety Administration, US Department of Transportation, Washington, DC, USA, 2005.

[23] X. Xiang, W. Qin, and B. Xiang, "Research on a DSRCbased rear-end collision warning model," IEEE Transactions on Intelligent Transportation Systems, vol. 15, no. 3, pp. 1054-1065, 2014.

[24] X. Qu and S. Wang, "Long-distance-commuter (LDC) lane: a new concept for freeway traffic management," Computer-Aided Civil and Infrastructure Engineering, vol. 30, no. 10, pp. 815-823, 2015.

[25] Y. Kuang, X. Qu, and S. Wang, "A tree-structured crash surrogate measure for freeways," Accident Analysis \& Prevention, vol. 77, pp. 137-148, 2015.

[26] X. Qu, S. Wang, and J. Zhang, “On the fundamental diagram for freeway traffic: a novel calibration approach for single-regime models," Transportation Research Part B: Methodological, vol. 73, pp. 91-102, 2015.

[27] X. Qu, Y. Yang, Z. Liu, S. Jin, and J. Weng, "Potential crash risks of expressway on-ramps and off-ramps: a case study in Beijing, China," Safety Science, vol. 70, pp. 58-62, 2014.

[28] L. Du, L. Han, and S. Chen, "Coordinated online in-vehicle routing balancing user optimality and system optimality through information perturbation," Transportation Research Part B: Methodological, vol. 79, pp. 121-133, 2015.

[29] P. G. Gipps, "A behavioural car-following model for computer simulation," Transportation Research B, vol. 15, no. 2, pp. 105111, 1981. 


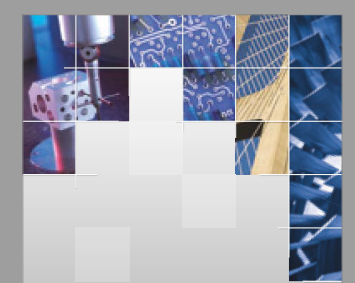

\section{Enfincering}
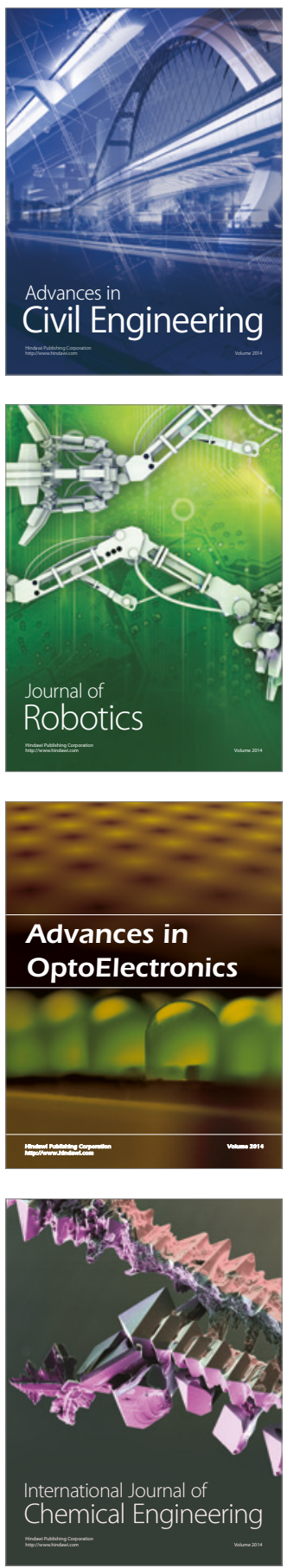

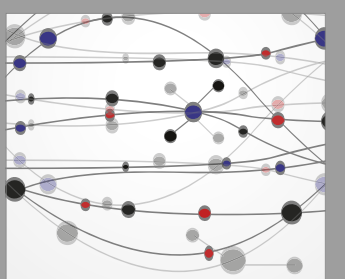

The Scientific World Journal

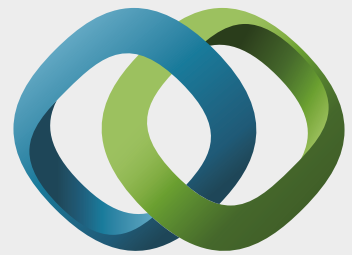

\section{Hindawi}

Submit your manuscripts at

https://www.hindawi.com
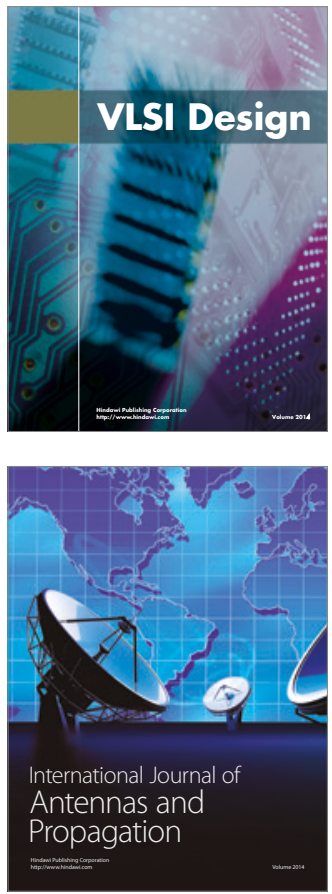

\section{Rotating}

Machinery
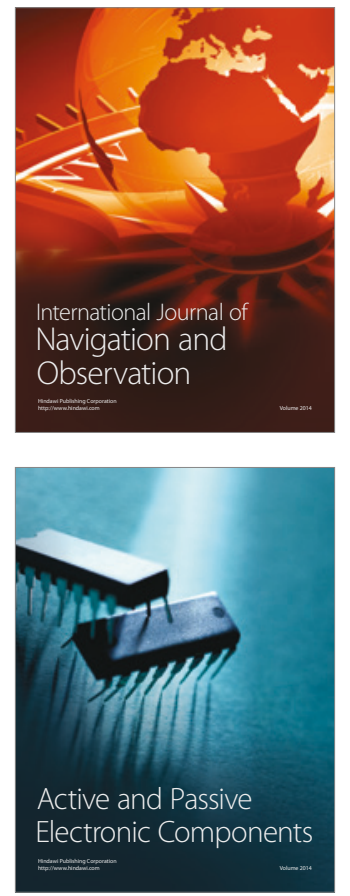
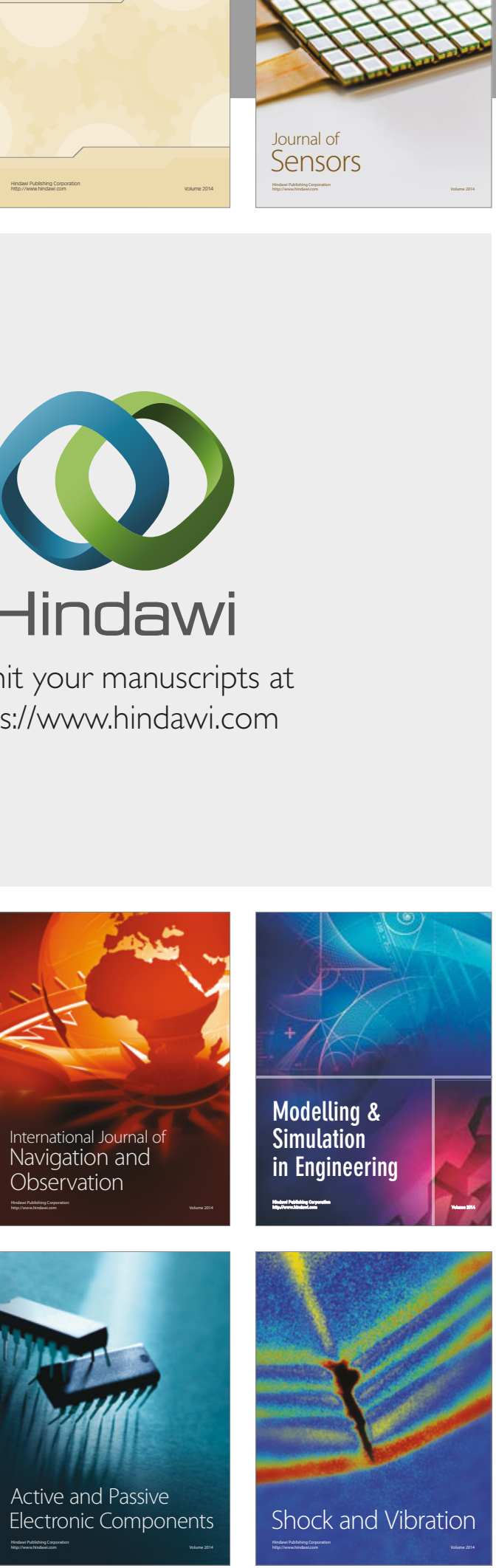
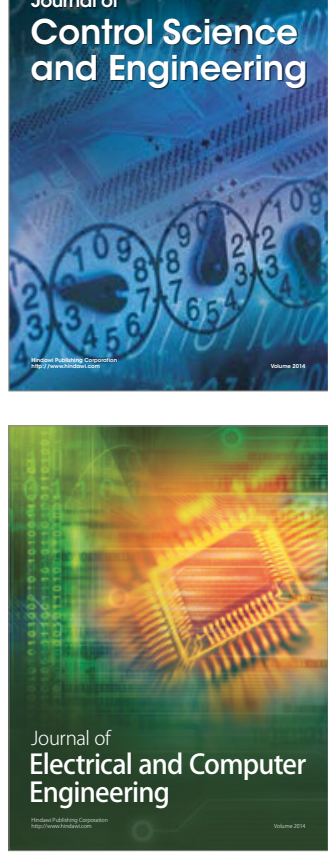

Distributed

Journal of

Control Science

and Engineering
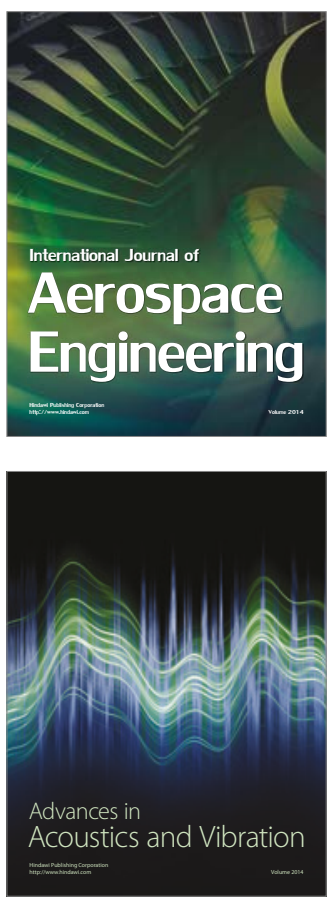

Sensor Networks 